\title{
CRECIMIENTO Y CONTENIDO DE CLOROFILA DEL MAGUEY MEZCALERO (Agave potatorum Zucc.) EN POLICULTIVO CON MAÍZ Y FRIJOL
}

\author{
GROWTH AND CHLOROPHYLL CONTENT OF Agave potatorum Zucc. \\ INTERCROPPED WITH CORN AND BEAN
}

\author{
Saúl Martínez-Ramírez ${ }^{1 *}$, Gilberto Bautista-Sánchez ${ }^{1}$, Eucebio C. Pedro-Santos ${ }^{1}$ y Pedro D. Guerrero-Cruz ${ }^{2}$
}

\begin{abstract}
${ }^{1}$ Universidad Tecnológica de la Mixteca. Km 2.5 Carr. Huajuapan-Acatlima. 69000, Huajuapan de León, Oaxaca, México. Tel. 01 (953) 53 22933 Ext. 550 y Fax 01(953) 53 20214. ${ }^{2}$ Brigada de Educación para el Desarrollo Rural 15, Dirección General de Educación Tecnológica Agropecuaria, Secretaría de Educación Pública. Valerio Trujano 1, Palacio Municipal. 69000, Huajuapan de León, Oaxaca, México.

${ }^{*}$ Autor para correspondencia (saulmr@mixteco.utm.mx)
\end{abstract}

\section{RESUMEN}

Se evaluó el crecimiento del maguey mezcalero (Agave potatorum Zucc.) cultivado en asociación con maíz (Zea mays L.) y frijol (Phaseolus vulgaris L.), en Huajuapan de León, Oaxaca, México. Se establecieron tres tratamientos: 1. Maguey en monocultivo, 2. Maguey con maíz y 3. Maguey con frijol. Cada tratamiento se repitió seis veces. Se usó un diseño experimental de bloques completos con tratamientos aleatorizados. Se empleó un maguey como unidad experimental. De agosto de 2011 a julio de 2012 se registraron mensualmente las nuevas hojas (desplegadas) y el área foliar. Estas variables se evaluaron cada mes y su cantidad acumulada mes a mes. Al final del estudio se evaluó: diámetro de planta, concentración de clorofila en hojas y contenido de humedad en el suelo. Se realizó un análisis de varianza y prueba de Tukey $(P=0.05)$. La evaluación mensual mostró que en agosto de 2011 los magueyes con frijol desplegaron 42 y $12 \%$ más hojas que los magueyes en monocultivo y con maíz, respectivamente; en cambio para febrero de 2012 desplegaron 100 y $\mathbf{5 4} \%$ más hojas que los magueyes con maíz y en monocultivo, respectivamente. La suma mes a mes mostró que de octubre de 2011 a julio de 2012 los magueyes con frijol tuvieron más hojas que los asociados con maíz, la máxima diferencia, de $28 \%$, ocurrió en mayo; de enero a junio de 2012 los magueyes con frijol exhibieron más área foliar que los asociados con maíz, la máxima diferencia, de $49 \%$, ocurrió en mayo. La evaluación anual mostró que los magueyes con frijol desplegaron $36 \%$ más hojas que los magueyes en monocultivo. La humedad del suelo no varió significativamente entre tratamientos. Se concluyó que el maguey mezcalero crece más cuando se asocia con frijol y menos cuando se asocia con maíz.

Palabras clave: Agave potatorum, asociación de cultivos, crecimiento.

\section{SUMMARY}

Growth of Agave potatorum Zucc. intercropped with corn (Zea mays L.) and bean (Phaseolus vulgaris L.) was assessed in Huajuapan de León, Oaxaca. Three treatments were established: 1 . Maguey in monoculture; 2. Maguey intercropped with corn; and 3. Maguey intercropped with bean. Every treatment was replicated six times. An experimental design of randomized complete blocks was used. One agave plant was one experimental unit. Monthly, from August 2011 to July 2012, number of new unfolded leaves and leaf area were recorded. These variables by month and accumulated month to month were analyzed. To the end of the study, increments in diameter, chlorophyll content in leaves and moisture in soil were evaluated. Data were submitted to analysis of variance and mean comparisons by Tukey's test with $P$ $=0.05$. Monthly evaluation showed than in August 2011 the agaves intercropped with bean unfolded 42 and $12 \%$ more leaves than agaves in monoculture and those intercropped with corn, respectively; in February 2102, agave plants unfolded 100 and $54 \%$ more leaves than agaves intercropped with corn or under monoculture, respectively. Month to month accumulation showed that from October 2011 to July 2012, agaves intercropped with bean formed more leaves than agaves intercropped with corn, with a maximum difference of $28 \%$ in May; from January to June 2012, it showed more leaf area, with a maximum difference of $49 \%$ in May. Annual evaluation showed that agaves plants intercropped with bean unfolded $36 \%$ more leaves than those in monoculture. Moisture of soil did not change significantly between treatments. It was concluded that agave mezcalero can grow more when intercropped with bean, and it grows less when intercropped with corn.

Index words: Agave potatorum, intercrops, growth.

\section{INTRODUCCIÓN}

El crecimiento de las especies vegetales es afectado por su interacción con el ambiente abiótico y biótico. Entre éstas, son importantes la competencia y la facilitación o complementación que ocurre entre especies (Soliveres et al., 2008). La sostenibilidad de los agroecosistemas se basa en procesos ecológicos naturales que ocurren por la diversidad de especies. Los policultivos son sistemas que tratan de imitar procesos naturales y se caracterizan porque dos o más especies son plantadas espacialmente próximas, con la consecuente competencia o complementación entre ellas (Vandermeer, 1992); con estos sistemas se pretende obtener mayores beneficios económicos (Rodríguez et al., 2008).

La productividad de los policultivos es mayor que la de los monocultivos sometidos al mismo nivel de manejo. Las ganancias en rendimiento varían de 20 a $60 \%$ y son acumulables a través del tiempo, ya que los policultivos tienen menor incidencia de plagas y usan con mayor eficiencia los nutrientes, el agua y la radiación solar (Lithourgidis et al., 2011). Olvera et al. (2000) encontraron que en 
suelos francos y en laderas de 40 a $60 \%$ de pendiente, la asociación de Agave angustifolia Haw. con maíz (Zea mays L.) elevó el pH del suelo de 5.4 a 6.6 y el contenido de fósforo y potasio en más de $100 \%$, y en terrenos con pendientes de $12 \%$ el contenido de materia orgánica se incrementó en $99 \%$.

Según Sánchez (2005), en los Distritos de Tlacolula, Ejutla, Ocotlán y Miahuatlán del Estado de Oaxaca se practica la siembra semi-intensiva y extensiva de A. angustifolia asociada con cultivos anuales, con el objetivo de mejorar las condiciones del suelo, especialmente en la captación y retención de humedad, reducción de plagas y enfermedades, y mejorar la cosecha de los cultivos anuales. Aunado a estos beneficios, por tener el metabolismo ácido de las crasuláceas (MAC) el maguey usa con mayor eficiencia el agua, lo cual puede ser una ventaja durante los periodos de sequía (Nobel, 1991), capacidad que debería aprovecharse más. Borland et al. (2009) reportaron incrementos sustanciales de biomasa para plantas MAC crecidas en tierras marginales y bajo elevadas concentraciones de $\mathrm{CO}_{2}$, por lo que podrían jugar un papel importante en la captura de $\mathrm{CO}_{2}$ atmosférico en regiones áridas y semiáridas del mundo.

Entre la biodiversidad de la Mixteca oaxaqueña se encuentran varias especies de maguey, mismas que los pueblos indígenas y mestizos han aprovechado como fuente de alimento, bebida, medicina, combustible, cobijo, ornato, fibras duras extraídas de las hojas ("ixtle"), abono, construcción de viviendas y elaboración de implementos agrícolas. Su empleo como alimentos y fibra pervive desde hace siete mil años (García-Mendoza, 2007), aunque Domínguez et al. (2008) refieren que su uso como alimento en Mesoamérica data de hace por lo menos nueve mil años.

La concentración de azúcares en A. potatorum cultivado en tierras marginales de la Mixteca Baja de Oaxaca (Martínez et al., 2012) hacen que sea una especie estratégica para cultivarse de manera comercial en las condiciones edafoclimáticas de la región. Con los factores que limitan la producción de los cultivos agrícolas y las características metabólicas de los magueyes, éstos podrían alcanzar una productividad comparable a la de especies agrícolas $\mathrm{C}_{3} \mathrm{y}$ $C_{4}$. Según Nobel (1991), algunas plantas MAC cultivadas pueden alcanzar a producir $47 \mathrm{Mg} \mathrm{ha}^{-1} \mathrm{año}^{-1}$, en comparación con las plantas $\mathrm{C}_{3}$ que producen hasta $39 \mathrm{Mg} \mathrm{ha}^{-1}$ año ${ }^{-1}$ y con las plantas $\mathrm{C}_{4}$ que alcanzan los $49 \mathrm{Mg} \mathrm{ha}^{-1} \mathrm{anoo}^{-1}$. Sin embargo, la desventaja del cultivo de A. potatorum es el prolongado tiempo de maduración pues el productor debe esperar 7 años para obtener ingresos económicos.

Una estrategia para obtener ingresos en la misma parcela y en periodos más cortos, es cultivar el maguey en asociación con especies agrícolas de ciclo anual, como ya se realiza en Valles Centrales de Oaxaca con A. angustifolia (SAGARPA, 2004). Con base en lo anterior, el objetivo de esta investigación fue evaluar el crecimiento del maguey mezcalero (A. potatorum) en asociación con maíz y con frijol (Phaseolus vulgaris L.) en condiciones de temporal (secano) en la Mixteca oaxaqueña.

\section{MATERIALES Y MÉTODOS}

El experimento efectuado para evaluar el crecimiento del maguey mezcalero en asociación con maíz y con frijol se inició en junio de 2011 en la comunidad de Rancho Dolores, que se sitúa a $6 \mathrm{~km}$ al norte de Huajuapan de León, Oaxaca, México. El clima del sitio es semiárido-cálido con un periodo de lluvias de junio a septiembre, con una precipitación de 500 a $800 \mathrm{~mm}$ y temperatura media anual de 18 a $22{ }^{\circ} \mathrm{C}($ Trejo, 2004). La vegetación natural corresponde a la selva baja caducifolia (Torres, 2004). El suelo es un Litosol, somero, pedregoso, cuyo uso puede ser limitado por la carencia de agua suficiente y la erosión, se desarrolla sobre un sustrato geológico de rocas metamórficas o calizas, en ocasiones expuestas (Alfaro, 2004).

Se evaluaron tres tratamientos: 1 . Maguey en monocultivo; 2. Maguey asociado con maíz criollo destinado para elaboración de pozole; y 3 . Maguey asociado con un frijol "negro" de la región. Los tratamientos se establecieron en un diseño experimental en bloques completos con tratamientos aleatorizados, con seis repeticiones, en un terreno marginal que no había sido cultivado durante 2 años. El suelo se roturó y surcó con arado de discos tirado por tractor agrícola. La distancia entre surcos fue de $0.80 \mathrm{~m}$. Cada repetición consistió de cinco surcos de $16 \mathrm{~m}$ de largo. La unidad experimental fue una planta de maguey de 1 año de edad.

Los magueyes se plantaron con su cepellón, el 10 de junio de 2011; se registró la cantidad de hojas (todos los magueyes tenían 10 hojas), el diámetro de roseta y el área foliar. En monocultivo se plantó a una distancia de $1 \mathrm{~m}$ entre magueyes y a $1.6 \mathrm{~m}$ entre surcos, lo cual proporcionó una densidad de 6200 magueyes ha ${ }^{-1}$. En los policultivos se plantó en el fondo del surco central y a una distancia de $1 \mathrm{~m}$ entre magueyes, lo cual proporcionó una densidad de 2500 magueyes ha ${ }^{-1}$. El maíz se sembró el 10 de julio de 2011, en el fondo del surco en grupos de cuatro semillas. La distancia entre grupos de cuatro plantas de maíz fue de 60 $\mathrm{cm}$; se obtuvieron 83,000 plantas ha-1 ${ }^{-1}$ El frijol se sembró el 10 de julio de 2011 en la costilla del surco, en grupos de dos semillas; se obtuvieron 100,000 plantas $\mathrm{ha}^{-1}$. Los tres tratamientos no fueron fertilizados.

Como respuesta de los tratamientos se evaluaron las variables: hojas nuevas (desplegadas), área foliar, diámetro de roseta, contenido de clorofila en hoja y contenido de humedad 
en el suelo. Las dos primeras se registraron mensualmente de agosto de 2011 a julio de 2012, las tres últimas se midieron sólo en octubre de 2012, dos semanas después de ocurrida la última lluvia del año. Para registrar la cantidad mensual de hojas nuevas se marcó con tinta indeleble la última hoja desplegada del maguey al momento de plantarlo.

Cada mes, a partir de agosto de 2011, se registraron las hojas que se desplegaron después de la hoja marcada, mismas que se numeraron. La cantidad de hojas desplegadas y el área foliar se analizaron por mes y sus valores acumulados mes a mes durante 12 meses. En los valores acumulados se incluyó la cantidad de hojas y área foliar que tenían los magueyes al momento de su plantación en campo. Para medir el diámetro de roseta y estimar el incremento en área foliar se siguió el procedimiento descrito en Martínez et al. (2012). El contenido de clorofila, en unidades Spectrum, se registró con un medidor marca FieldScout (FS), modelo CM1000 ${ }^{\circledR}$ (Illinois, USA), las lecturas se hicieron en la parte media del haz en las cinco últimas hojas desplegadas de seis magueyes por bloque. El promedio de lecturas fue el contenido de clorofila de las plantas en cada bloque.

Para evaluar el contenido volumétrico del agua en el suelo se empleó un medidor marca FieldScout modelo TDR $300 \circledR$ (Illinois, USA) con barras de $12 \mathrm{~cm}$. Los sensores se insertaron en el suelo en el límite de la proyección vertical de las hojas más extendidas de seis magueyes por bloque, y se tomó una lectura por punto cardinal. El promedio de las 24 lecturas fue el contenido de agua del suelo en cada bloque. Al inicio del experimento se determinaron las siguientes variable edáficas de acuerdo a la NOM-021-RECNAT-2000 (Diario Oficial de la Federación, 2002): pH (8.2), conductividad eléctrica $\left(0.308 \mathrm{dS} \mathrm{m}^{-1}\right)$, P-Olsen (3.5 $\left.\mathrm{mg} \mathrm{kg}{ }^{-1}\right)$, densidad aparente $\left(1.55 \mathrm{~g} \mathrm{~cm}^{-3}\right)$, materia orgánica (2\%), proporción de arena, limo y arcilla (45, 35.5 y 19.5 \%). El análisis de varianza y prueba de Tukey con $\mathrm{P}=0.05$, para las variables de respuesta, se realizó con el programa Minitab $16 \circledR$.

\section{RESULTADOS Y DISCUSIÓN}

El análisis de varianza para la cantidad mensual de hojas desplegadas en agosto de 2011 y febrero de 2012 reveló diferencias significativas $(\mathrm{P}<0.05)$ entre tratamientos (Cuadro 1). Para el crecimiento mensual en área foliar no se detectaron diferencias significativas $(\mathrm{P}<0.05)$ en ninguno de los meses que duró el experimento (Cuadro 2). Con los datos tomados al final del estudio (julio de 2012), la prueba de medias indicó que no hubo diferencia significativa entre tratamientos en incremento en diámetro de roseta ni en área foliar $(\mathrm{P} \leq 0.05)$, pero sí en cantidad de hojas acumuladas y en contenido de clorofila (Cuadro 3).

Así, en la cantidad acumulada de hojas desplegadas mes a mes, el análisis estadístico mostró diferencias en 11 de los 12 meses que duró el experimento, con excepción de septiembre de 2011 (Cuadro 4); mientras que el área foliar mostró

Cuadro 1. Promedios $(n=6)$ mensuales de nuevas hojas desplegadas en cada tratamiento por Agave potatorum crecido en monocultivo y asociado con maíz o frijol. Huajuapan de León, Oaxaca.

\begin{tabular}{|c|c|c|c|c|c|c|}
\hline \multirow{2}{*}{ Año } & \multirow{2}{*}{ Mes } & \multicolumn{3}{|c|}{ Tratamientos } & \multirow{2}{*}{ DMSH } & \multirow{2}{*}{ Valor de F } \\
\hline & & Maguey & Maguey-maíz & Maguey-frijol & & \\
\hline \multirow{6}{*}{2011} & Junio & 10.0 & 10.0 & 10.0 & & \\
\hline & Agosto & $3.3 \mathrm{~b}$ & $4.2 \mathrm{a}$ & $4.7 \mathrm{a}$ & 1.1 & 0.01 \\
\hline & Septiembre & 2.8 & 2.3 & 3.2 & 1.5 & 0.36 \\
\hline & Octubre & 3.0 & 1.8 & 3.2 & 1.5 & 0.59 \\
\hline & Noviembre & 1.7 & 1.2 & 2.0 & 1.1 & 0.15 \\
\hline & Diciembre & 0.8 & 1.2 & 1.3 & 1.2 & 0.54 \\
\hline \multirow{7}{*}{2012} & Enero & 1.2 & 1.0 & 1.7 & 1.1 & 0.25 \\
\hline & Febrero & $1.3 \mathrm{ab}$ & $1.0 \mathrm{~b}$ & $2.0 \mathrm{a}$ & 0.9 & 0.04 \\
\hline & Marzo & 1.7 & 1.2 & 1.5 & 1.1 & 0.46 \\
\hline & Abril & 1.2 & 1.2 & 2.0 & 1.4 & 0.20 \\
\hline & Mayo & 1.0 & 1.0 & 1.8 & 1.5 & 0.25 \\
\hline & Junio & 2.0 & 2.5 & 2.8 & 1.6 & 0.40 \\
\hline & Julio & 2.5 & 4.7 & 4.8 & 2.9 & 0.15 \\
\hline
\end{tabular}

Medias con letras iguales o sin letras en la misma hilera no son estadísticamente diferentes (Tukey, 0.05). DMSH = diferencia mínima significativa honesta. 
Cuadro 2. Promedio mensual de área foliar $\left(\mathrm{cm}^{2}\right)$ en cada tratamiento de asociación por Agave potatorum crecido en monocultivo y asociado con maíz o frijol. Huajuapan de León, Oaxaca.

\begin{tabular}{|c|c|c|c|c|c|c|}
\hline \multirow{2}{*}{ Año } & \multirow{2}{*}{ Mes } & \multicolumn{3}{|c|}{ Tratamientos } & \multirow{2}{*}{ DMSH } & \multirow{2}{*}{ Valor de $\mathrm{F}$} \\
\hline & & Maguey & Maguey-maíz & Maguey-frijol & & \\
\hline \multirow{7}{*}{2011} & Junio & 363.7 & 301.4 & 343.8 & 68.9 & 0.69 \\
\hline & Agosto & 262.2 & 254.3 & 307.5 & 112.7 & 0.41 \\
\hline & Septiembre & 178.2 & 162.0 & 188.7 & 129.9 & 0.86 \\
\hline & Octubre & 227.0 & 124.8 & 216.5 & 103.0 & 0.04 \\
\hline & Noviembre & 91.5 & 84.4 & 145.2 & 77.8 & 0.13 \\
\hline & Diciembre & 38.3 & 75.5 & 80.9 & 63.9 & 0.19 \\
\hline & Enero & 88.2 & 66.9 & 131.6 & 86.8 & 0.16 \\
\hline \multirow{6}{*}{2012} & Febrero & 111.4 & 112.8 & 144.5 & 73.2 & 0.41 \\
\hline & Marzo & 144.5 & 91.7 & 121.4 & 75.7 & 0.21 \\
\hline & Abril & 96.3 & 89.7 & 181.5 & 122.3 & 0.12 \\
\hline & Mayo & 81.2 & 55.6 & 153.1 & 109.7 & 0.09 \\
\hline & Junio & 170.4 & 186.2 & 209.2 & 132.4 & 0.73 \\
\hline & Julio & 241.6 & 328.1 & 337.0 & 173.8 & 0.30 \\
\hline
\end{tabular}

Medias con letras iguales o sin letras en la misma hilera no son estadísticamente diferentes (Tukey, 0.05). DMSH = diferencia mínima significativa honesta.

Cuadro 3. Promedios $(n=6)$ de crecimiento final de Agave potatorum crecido en tres tratamientos de asociación (monocultivo, asociado con maíz y asociado con frijol), en Huajuapan de León, Oaxaca.

\begin{tabular}{|c|c|c|c|c|c|c|}
\hline \multirow{2}{*}{ Tratamiento } & $\mathrm{D}^{\dagger}$ & $\mathrm{D}$ & $\mathrm{AF}$ & \multirow{2}{*}{$\mathrm{HD}$} & C & HS \\
\hline & $(\mathrm{cm})$ & $(\mathrm{cm})$ & $\left(\mathrm{cm}^{2}\right)$ & & (US) & (US) \\
\hline Maguey en monocultivo & $23.5 \mathrm{a}$ & $33.0 \mathrm{a}$ & $1731 \mathrm{a}$ & $22.5 \mathrm{~b}$ & $332.7 \mathrm{~b}$ & $55.8 \mathrm{a}$ \\
\hline Maguey asociado con maíz & $24.8 \mathrm{a}$ & $36.3 \mathrm{a}$ & $1632 \mathrm{a}$ & $23.2 \mathrm{ab}$ & $315.5 \mathrm{~b}$ & $52.2 \mathrm{a}$ \\
\hline Maguey asociado con frijol & $24.3 \mathrm{a}$ & $43.3 \mathrm{a}$ & 2217 a & $30.5 \mathrm{a}$ & $384.3 \mathrm{a}$ & $51.9 \mathrm{a}$ \\
\hline DMSH & 2.7 & 10.4 & 602 & 7.5 & 33.9 & 13.0 \\
\hline Valor de F & 0.75 & 0.06 & 0.05 & 0.03 & $<0.01$ & 0.67 \\
\hline
\end{tabular}

Medias con letras iguales en la misma columna no son estadísticamente diferentes (Tukey, 0.05); $\mathrm{D}^{\dagger}=$ diámetro de planta al momento de la plantación en campo; $\mathrm{D}=$ crecimiento en diámetro; $\mathrm{AF}=$ crecimiento en área foliar; $\mathrm{HD}=$ crecimiento en cantidad de hojas desplegadas; $\mathrm{CC}=$ crecimiento en contenido de clorofila; HS = crecimiento en humedad de suelo; US = unidades Spectrum; DMSH = diferencia mínima significativa honesta.

diferencias solamente de enero a junio de 2012 (Cuadro 5). En la evaluación que se hizo al final del periodo de estudio se encontraron diferencias significativas $(\mathrm{P}<0.05)$ en cantidad de hojas desplegadas y en contenido de clorofila en hojas; en cambio, el contenido volumétrico de agua en el suelo no mostró diferencias significativas $(P>0.05)$ entre tratamientos.

Los magueyes que se cultivaron en asociación con frijol desplegaron 42 y $12 \%$ más hojas en agosto de 2011 que los magueyes en monocultivo y que los intercalados con maíz; en cambio, para febrero de 2012 habían desplegado 100 y $54 \%$ más hojas que los magueyes desarrollados en asociación con maíz y en monocultivo (Cuadro 1). Esta tendencia se mantuvo hasta el final del periodo de estudio. En este caso los magueyes que crecieron en asociación con frijol desplegaron 36 y $31 \%$ más hojas que los que crecieron en monocultivo y con maíz (Cuadro 3 ).

La cantidad acumulada de hojas mes a mes alcanzó su máxima diferencia (28 \%) en mayo de 2012, entre los magueyes que se cultivaron en asociación con frijol y los magueyes que se cultivaron en asociación con maíz, y en julio de 2012 (26\%) entre magueyes que se cultivaron en asociación con frijol y magueyes en monocultivo (Cuadro 4 y Figura 1). El área foliar acumulada mes a mes alcanzó su máxima diferencia ( $49 \%$ ) entre los magueyes que se cultivaron en asociación con frijol y los magueyes que se 
Cuadro 4. Suma de hojas desplegadas mes a mes por Agave potatorum crecido en monocultivo y asociado con maíz o frijol. Huajuapan de León, Oaxaca.

\begin{tabular}{|c|c|c|c|c|c|c|c|c|}
\hline \multirow{2}{*}{ Año } & \multirow{2}{*}{ Mes } & \multicolumn{3}{|c|}{ Tratamientos } & \multirow{2}{*}{ DMSH } & \multirow{2}{*}{ Valor de F } & \multicolumn{2}{|c|}{ Diferencia (\%) } \\
\hline & & $\mathrm{A}$ & $\mathrm{B}$ & $\mathrm{C}$ & & & C-A & C-B \\
\hline \multirow{3}{*}{2011} & Agosto & $13.3 \mathrm{~b}$ & $14.2 \mathrm{ab}$ & $14.7 \mathrm{a}$ & 1.1 & 0.03 & 10.0 & 3.5 \\
\hline & Octubre & $19.2 \mathrm{ab}$ & $18.3 \mathrm{~b}$ & $21.0 \mathrm{a}$ & 2.4 & 0.04 & 9.6 & 14.5 \\
\hline & Noviembre & $20.8 \mathrm{ab}$ & $19.5 \mathrm{~b}$ & $23.0 \mathrm{a}$ & 3.2 & 0.04 & 10.4 & 17.9 \\
\hline \multirow[b]{4}{*}{2012} & Enero & $22.8 \mathrm{ab}$ & $21.7 \mathrm{~b}$ & $26.0 \mathrm{a}$ & 3.4 & 0.01 & 13.9 & 20.0 \\
\hline & Febrero & $24.2 \mathrm{~b}$ & $22.7 \mathrm{~b}$ & $28.0 \mathrm{a}$ & 3.6 & 0.01 & 15.7 & 23.5 \\
\hline & Marzo & $25.8 \mathrm{~b}$ & $23.8 \mathrm{~b}$ & $29.5 \mathrm{a}$ & 3.3 & $<0.01$ & 14.2 & 23.8 \\
\hline & Abril & $27.0 \mathrm{~b}$ & $25.0 \mathrm{~b}$ & $31.5 \mathrm{a}$ & 3.5 & $<0.01$ & 16.7 & 26.0 \\
\hline
\end{tabular}

Medias sin letras o con letras iguales en la misma hilera no son estadísticamente diferentes (Tukey, 0.05); DMSH = diferencia mínima significativa honesta; A = maguey en monocultivo; $\mathrm{B}=$ maguey asociado con maíz; $\mathrm{C}=$ maguey asociado con frijol.

Cuadro 5. Suma de área foliar $\left(\mathrm{cm}^{2}\right)$ mes a mes generado por Agave potatorum crecido en monocultivo y asociado con maíz o frijol. Huajuapan de León, Oaxaca.

\begin{tabular}{|c|c|c|c|c|c|c|c|c|}
\hline \multirow{2}{*}{ Año } & \multirow{2}{*}{ Mes } & \multicolumn{3}{|c|}{ Tratamientos } & \multirow{2}{*}{ DMSH } & \multirow{2}{*}{ Valor de F } & \multicolumn{2}{|c|}{ Diferencia (\%) } \\
\hline & & A & B & $\mathrm{C}$ & & & C-A & $\mathrm{C}-\mathrm{B}$ \\
\hline \multirow{5}{*}{2011} & Agosto & 262 & 254 & 307 & 113 & 0.41 & 17.3 & 20.9 \\
\hline & Septiembre & 440 & 416 & 496 & 203 & 0.56 & 12.7 & 19.2 \\
\hline & Octubre & 667 & 541 & 713 & 250 & 0.20 & 6.8 & 31.7 \\
\hline & Noviembre & 759 & 625 & 858 & 278 & 0.12 & 13.0 & 37.2 \\
\hline & Diciembre & 797 & 701 & 939 & 261 & 0.09 & 17.8 & 33.9 \\
\hline \multirow{7}{*}{2012} & Enero & $885 \mathrm{ab}$ & $768 \mathrm{~b}$ & $1070 \mathrm{a}$ & 283 & 0.04 & 20.9 & 39.4 \\
\hline & Febrero & $997 \mathrm{ab}$ & $881 \mathrm{~b}$ & $1215 \mathrm{a}$ & 318 & 0.05 & 21.9 & 38.0 \\
\hline & Marzo & $1141 \mathrm{ab}$ & $972 \mathrm{~b}$ & $1336 \mathrm{a}$ & 331 & 0.04 & 17.1 & 37.4 \\
\hline & Abril & $1238 \mathrm{ab}$ & $1062 \mathrm{~b}$ & $1518 \mathrm{a}$ & 393 & 0.03 & 22.6 & 42.9 \\
\hline & Mayo & $1319 \mathrm{ab}$ & $1118 \mathrm{~b}$ & $1671 \mathrm{a}$ & 442 & 0.02 & 26.7 & 49.5 \\
\hline & Junio & $1489 \mathrm{ab}$ & $1304 \mathrm{~b}$ & $1880 \mathrm{a}$ & 498 & 0.03 & 26.2 & 44.2 \\
\hline & julio & 1731 & 1632 & 2217 & 602 & 0.05 & 28.1 & 35.9 \\
\hline
\end{tabular}

Medias sin letras o con letras iguales en la misma hilera no son estadísticamente diferentes (Tukey, 0.05); DMSH = diferencia mínima significativa honesta; $\mathrm{A}=$ maguey en monocultivo; $\mathrm{B}=$ maguey asociado con maíz; $\mathrm{C}=$ maguey asociado con frijol.

cultivaron en asociación con maíz, en mayo de 2012 (Cuadro 5 y Figura 2). En síntesis, $A$. potatorum creció más, en cantidad de hojas desplegadas y en área foliar, cuando se cultivó asociado con frijol que cuando creció en monocultivo o asociado con maíz.

El efecto positivo de la asociación con el frijol y el efec- to negativo de estar asociado con el maíz que mostró el maguey reflejan interacciones de complementación y de competencia, respectivamente. La competencia con el maíz probablemente se deba a que éste es muy demandante de nitrógeno, tal como lo comprobaron Améndola-Massiotti et al. (2011). Además, los magueyes jóvenes tienden a crecer menos cuando compiten con otras especies, tal como 
Monroy et al. (2005) observaron que sucedió con Agave tequilana Weber.

En contraste, el beneficio que tuvo A. potatorum al crecer en asociación con frijol probablemente es causado por el nitrógeno atmosférico que es fijado por el frijol, ya que generalmente las fabáceas proporcionan cantidades sustanciales de nitrógeno disponible para los cultivos (Havlin et al., 2005) y contribuyen con una parte importante del nitrógeno fijado anualmente en el ambiente terrestre (Taiz y Zeiger, 2006).

Existen evidencias que A. potatorum y otras especies del mismo género responden positivamente a la aplicación de dosis bajas de nitrógeno, de 40 a $50 \mathrm{~kg} \mathrm{ha}^{-1}$ (SAGARPA, 2004; Martínez et al., 2012). Se ha encontrado que los magueyes en etapa juvenil despliegan más hojas cuando se fertilizan con dosis bajas de nitrógeno. Díaz et al. (2011) encontraron que en tres meses de crecimiento las plantas jóvenes (con 3 a 4 hojas) de Agave cocui Trelease que se fertilizaron cada $15 \mathrm{~d}$ con 0.5 y $1 \mathrm{~g}$ de nitrato de amonio por planta desplegaron 8 hojas, en comparación con 7 hojas, respectivamente; en contraste, las plantas del testigo formaron 6 hojas por maguey. Mussett y Díaz (2001) observaron que $A$. cocui creció más en altura cuando estuvo asociado con Erythrina velutina Willd.
Otras especies como girasol (Helianthus annuus L. cv. 'Victoria') (Morales-Rosales et al., 2006), el maíz (Vélez et al., 2007) y el café (Coffea arabica L.) (Moreno, 2007), también han mostrado un efecto benéfico en su crecimiento cuando se cultivan asociados con frijol, lo cual indica una interacción de complementación y no de competencia. Este beneficio se atribuye, además de la aportación de nitrógeno del frijol, a una mayor eficiencia en el uso del agua y de la irradiación fotosintéticamente activa (Morales-Rosales et al., 2006).

El contenido de clorofila en unidades Spectrum fue 22 y $16 \%$ mayor en los magueyes asociados con frijol en comparación con la asociación con maíz y con el maguey monocultivado, respectivamente. Regularmente, al aumento en la fertilización nitrogenada corresponde un mayor contenido de clorofila foliar, como se ha encontrado en maíz (Rincón y Ligarreto, 2010), en orégano (Origanum vulgare L.) (Calderón et al., 2011), y tomate (Lycopersicum esculentum L.) (Rodríguez et al., 1998). Esta respuesta positiva en el contenido de clorofila en las hojas de A. potatorum probablemente se deba al aumento en la disponibilidad de nitrógeno edáfico ocasionado por el frijol.

No se observaron diferencias significativas en la humedad edáfica cuando el maguey creció en monocultivo o asociado con maíz o frijol, lo cual sugiere que el agua no

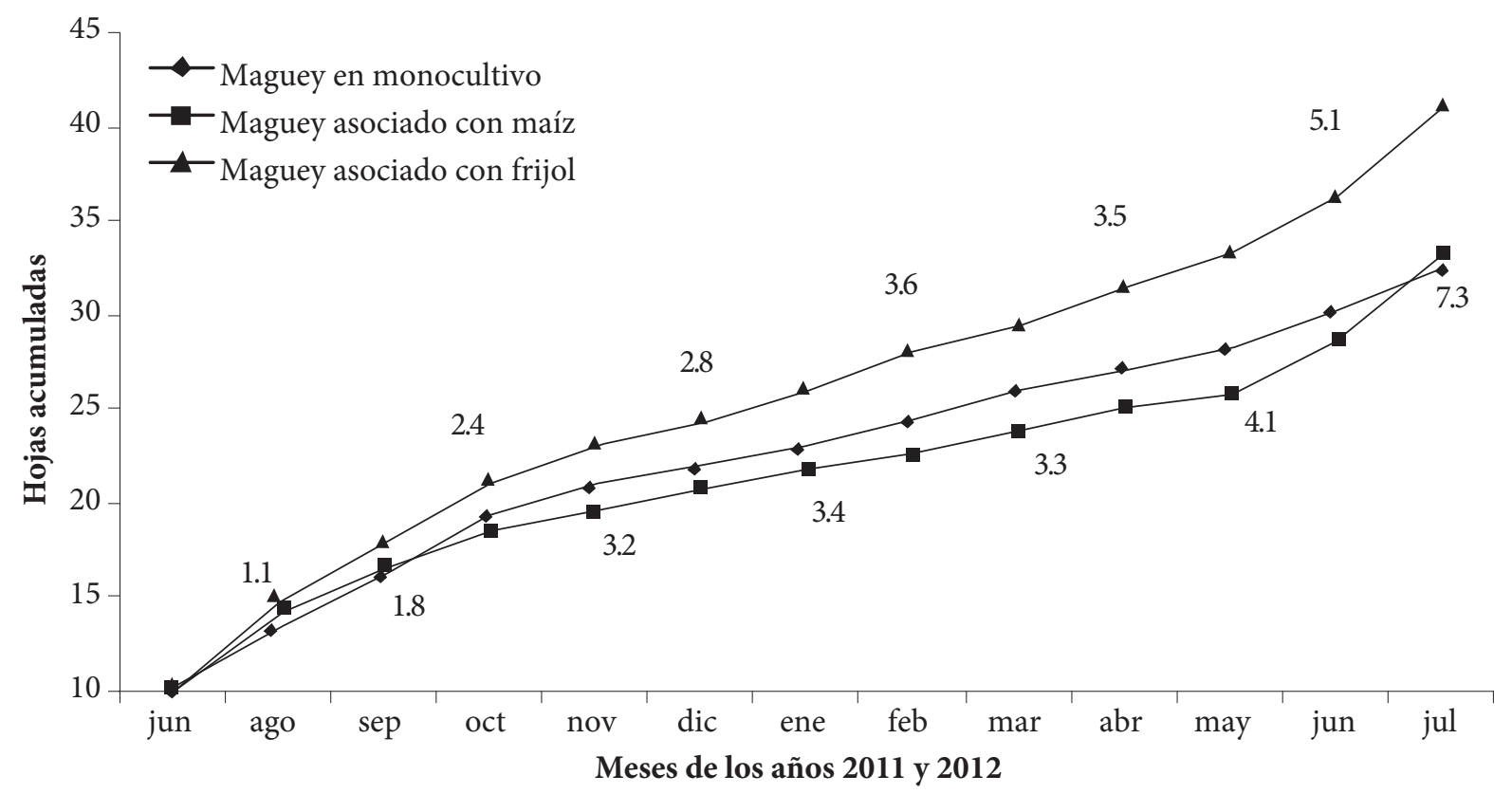

Figura 1. Suma de las hojas desplegadas mes a mes por Agave potatorum crecido en monocultivo y asociado con maíz o frijol. Huajuapan de León, Oaxaca. Los niveles de error variaron desde $<0.01$ hasta 0.04 , con excepción de septiembre de 2011 en el que fue de 0.07. Las cifras junto a las líneas de tendencia corresponden a la diferencia mínima significativa honesta para cada mes. 


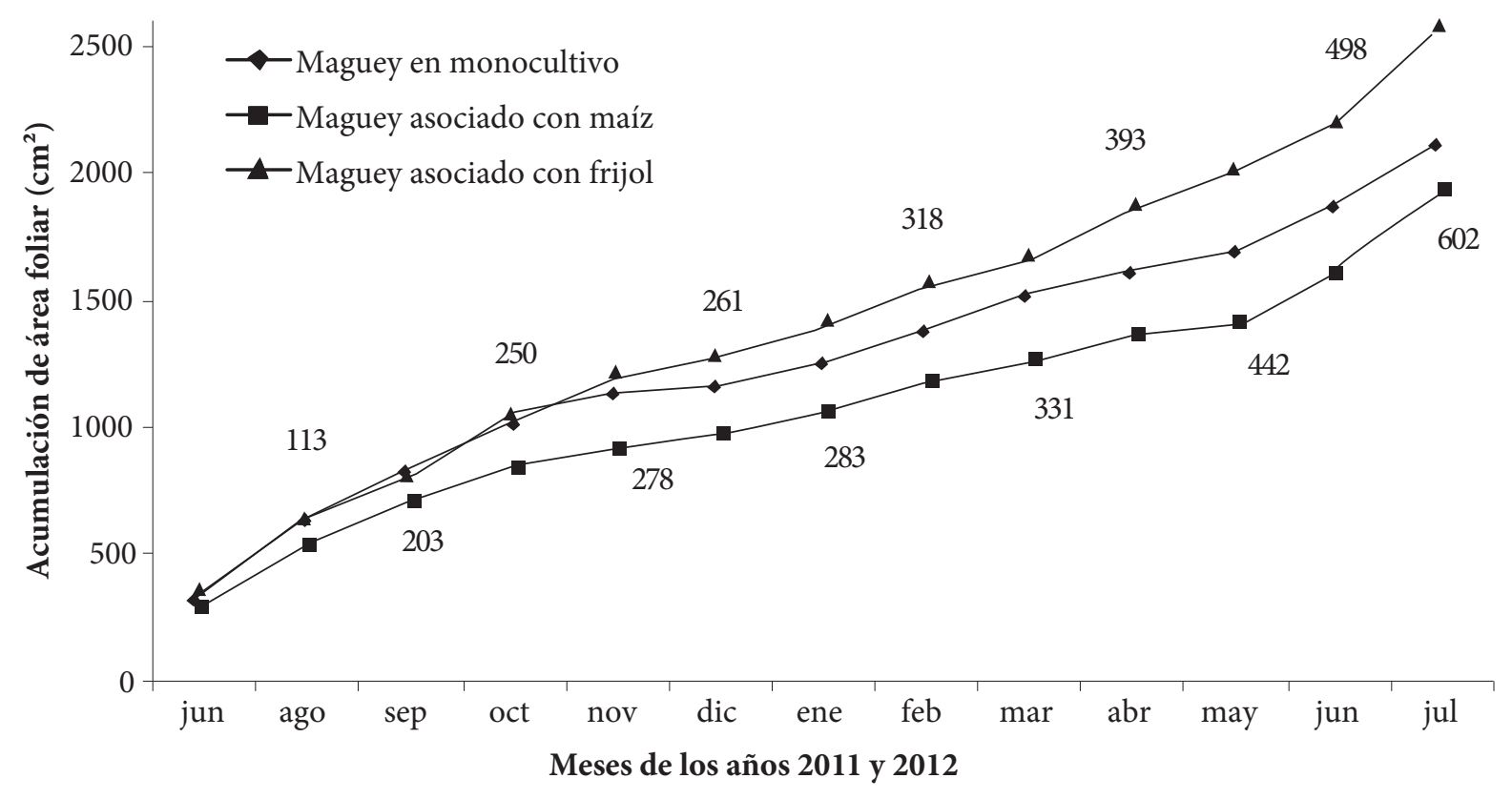

Figura 2. Suma de área foliar $\left(\mathrm{cm}^{2}\right)$ mes a mes generado por Agave potatorum crecido en monocultivo y asociado con maíz o frijol. Huajuapan de León, Oaxaca. Los niveles de error variaron desde 0.09 hasta 0.56 de agosto a diciembre de 2011 y desde 0.02 hasta 0.05 de enero a julio de 2012. Las cifras junto a las líneas de tendencia corresponden a la diferencia mínima significativa honesta para cada mes.

fue un factor limitante en estos tres tratamientos, aun cuando hubo competencia entre las plantas por la humedad del suelo. Se confirma el efecto benéfico de los policultivos en el uso eficiente del recurso ambiental agua (Lithourgidis et al., 2011), como también se detectó en la asociación maízfrijol (Ogindo y Walker, 2005). Según Sánchez (2005) A. angustifolia asociado con cultivos anuales mejora las condiciones del suelo, especialmente en la captación y retención de humedad.

\section{CONCLUSIONES}

El maguey mezcalero A. potatorum desplegó más hojas, desarrolló más área foliar y contenía más clorofila en hojas cuando se cultivó asociado con frijol comparado con el maguey en monocultivo. En contraste, la asociación con maíz redujo el crecimiento de este agave. El desarrollo de $A$. potatorum en monocultivo, asociado con maíz o con frijol no generó diferencias significativas en el contenido de humedad del suelo al final del periodo de estudio.

\section{AGRADECIMIENTOS}

Al Programa de Mejoramiento del Profesorado (PROMEP) y a la Universidad Tecnológica de la Mixteca, por el financiamiento de la investigación. A la comunidad de Rancho Dolores, especialmente al Sr. Ángel Guerrero Cruz, por el préstamo gratuito del terreno donde se realizó el experimento.

\section{BIBLIOGRAFÍA}

Alfaro S. G. (2004) Suelos. In: Biodiversidad de Oaxaca. A. J. García-Mendoza, M. J. Ordóñez y M. Briones-Salas (eds.). Ed. UNAMFondo Oaxaqueño para la Conservación de la NaturalezaWorld Wildlife Fundation. México. pp:55-65.

Améndola-Massiotti R., I. Cach-Gómez, E. Âlvarez-Sánchez, I. LópezCruz, J. Burgueño-Ferreira, P. Martínez-Hernández y D. Cristóbal-Acevedo (2011) Balance de nitrógeno en maíz forrajero con diferente fertilización y fase de rotación con praderas. Agrociencia 45:177-193.

Borland A. N., H. Griffits, J. Hartwell and J. A. C. Smith (2009) Exploiting the potential of plants with crassulacean acid metabolism for bioenergy production on marginal lands. Journal of Experimental Botany 60:2879-2896.

Calderón M. L. A., A. M. Bernal y M. M. Pérez (2011) Ensayo preliminar sobre la utilización de un medidor portátil de clorofila para estimar el nitrógeno foliar en orégano (Origanum vulgare L.). Revista Facultad de Ciencias Básicas 7:150-165.

Diario Oficial de la Federación (2002) Norma Oficial Mexicana NOM021-RECNAT-2000 que establece las especificaciones de fertilidad, salinidad y clasificación de suelos. Estudios, muestreo y análisis. Diario Oficial de la Federación del 31 de diciembre de 2002. http://www.profepa.gob.mx/innovaportal/file/3335/1/ nom-021-semarnat-2000.pdf (Junio 2011).

Díaz J. G., G. Rojas, Y. Him, N. Hernández, E. Torrealba y Z. Rodríguez (2011) Efecto de la fertilización nitrogenada sobre el crecimiento en vivero de Cocuy (Agave cocui Trelease) Revista de la Facultad de Agronomía Luz (Supl. 1) 28:264-472.

Domínguez R. M. S., M. de la L. González J., C. Rosales, C. Quiñones, S. Delgadillo, S. J. Mireles y E. Pérez (2008) El cultivo in vitro como herramienta para el aprovechamiento, mejoramiento 
y conservación de especies del género Agave. Investigación y Ciencia 41:53-62.

García-Mendoza A. J. (2007) Los agaves de México. Ciencias 87:14-23.

Havlin J. L., J. D. Beaton, S. L. Tisdale and W. L. Nelson (2005) Soil fertility and fertilizers. An Introduction to Nutrient Management. Ed. Pearson Prentice Hall. New Jersey, USA. 515 p.

Lithourgidis A. S., C. A. Dordas, C. A. Damalas and D. N. Vlachostergios (2011) Annual intercrops: an alternative pathway for sustainable agriculture. Australian Journal of Crop Science 5:396410.

Martínez R. S., A. Trinidad, C. Robles, A. Galvis, T. M. Hernández, J. A. Santizo, G. Bautista y E. C. Pedro (2012) Crecimiento y sólidos solubles de Agave potatorum Zucc. inducidos por riego y fertilización. Revista Fitotecnia Mexicana 35:61-68.

Monroy R. B., E. Pimienta, S. A. Hurtado y J. García (2005) Efecto competitivo de la maleza sobre el crecimiento y la respuesta fisiológica de agave (Agave tequilana Weber var. "azul") en la región de Tequila, Jalisco, México. Scientia-CUCBA 7:105-112.

Morales-Rosales E. J., J. A. Escalante-Estrada, L. Tijerina-Chávez, V. Volke-Haller y E. Sosa-Montes (2006) Biomasa, rendimiento, eficiencia en el uso del agua y de la radiación solar del agrosistema girasol-frijol. Terra Latinoamericana 24:55-64.

Moreno B. A. M. (2007) Productividad de café en sistemas intercalados. In: Sistemas de Producción de Café en Colombia. O. H. Ospina y S. M. Marín (eds.). Ed. Centro Nacional de Investigaciones de Café. Colombia. pp:255-274.

Mussett S. y M. Díaz (2001) Efecto de la densidad de siembra sobre el crecimiento de Agave cocui Trelease en asociación con Erythrina velutina bajo un sistema agroforestal simultáneo en las Zonas áridas del Edo Falcón. Croizatia 2:6-13.

Nobel P. S. (1991) Environmental productivity indices and productivity for Opuntia ficus-indica under current and elevated atmospheric $\mathrm{CO}_{2}$ levels. Plant Cell and Environment 14:637-646.

Ogindo H. O. and S. Walker (2005) Comparison of measured changes in seasonal soil water content by rained maize-bean intercrop and component cropping in semi arid region in South. Physics and Chemistry of the Earth 30:799-808.

Olvera S. M. D., W. Wruck, A. Reyes y H. G. Cortés (2000) Modelos tipo y beneficios de las líneas vegetativas intermedias en suelos de ladera. Investigación y Ciencia 41:53-62.
Rincón A. y G. A. Ligarreto (2010) Relación entre nitrógeno foliar y el contenido de clorofila, en maíz asociado con pastos en el Piedemonte Llanero colombiano. Revista Corpoica Ciencia y Tecnología Agropecuaria 11:122-128.

Rodríguez M. M., G. Alcántar, A. Aguilar, J. Etchevers y J. Santizo (1998) Estimación de la concentración de nitrógeno y cloro fila en tomate mediante un medidor portátil de clorofila. Terra 16:135-141.

Rodríguez H., L. Acosta, I. Hechevarría, M. Milanés y C. A. Rodríguez (2008) Estudio comparativo entre el monocultivo y la asociación de cultivo en varias plantas medicinales. Revista Cubana de Plantas Medicinales 13:1-7.

SAGARPA, Secretaría de Agricultura, Ganadería, Desarrollo Rural, Pesca y Alimentación (2004) Diagnóstico de la Cadena Productiva del Sistema Producto Maguey-Mezcal. Ed. SAGARPADelegación Oaxaca, Oaxaca, México. 213 p

Sánchez L. A. (2005) Oaxaca, Tierra de Maguey y Mezcal. Ed. Instituto Tecnológico de Oaxaca. Oaxaca, México. 235 p.

Soliveres C. S., P. García, A. del P. Castillo, A. Escudero, F. Valladares y F. T. Maestre (2008) Las interacciones planta-planta varían con el nivel de estrés abiótico: dos estudios de caso en clima semiárido. Cuadernos de la Sociedad Española de Ciencias Forestales 28:271-276.

Taiz L. and E. Zeiger (2006) Plant Physiology. Sinauer Associates, Inc. Publishers. Massachusetts, USA. 764 p.

Torres C. R. (2004) Tipos de vegetación. In: Biodiversidad de Oaxaca. A. J. García-Mendoza, M. J. Ordóñez y M. Briones-Salas (eds.). Ed. UNAM-Fondo Oaxaqueño para la Conservación de la Naturaleza-World Wildlife Fundation. México. pp:105-117.

Trejo I. (2004) Clima. In: Biodiversidad de Oaxaca. A. J. García-Mendoza, M. J. Ordóñez y M. Briones-Salas (eds.). Ed. UNAM-Fondo Oaxaqueño para la Conservación de la Naturaleza-World Wildlife Fundation. México. pp:67-85.

Vandermeer J. (1992) The Ecology of Intercropping. Cambridge University Press. Cambridge, UK. 237 p.

Vélez V. L. D., J. Clavijo y G. A. Ligarreto (2007) Análisis ecofisiológico del cultivo asociado maíz (Zea mays L)-frijol voluble (Phaseolus vulgaris L.). Revista Facultad Nacional de Agronomía-Medellín 60:3965-3984. 